\title{
Philos-type oscillation criteria for second-order linear impulsive differential equation with damping
}

\author{
Kunwen Wen ${ }^{1}$, Yuping Zeng ${ }^{1}$, Huaqin Peng ${ }^{2}$ and Lifang Huang ${ }^{3 *}$
}

\section{"Correspondence:}

hlfang208@163.com

${ }^{3}$ School of Statistics and

Mathematics, Guangdong

University of Finance and

Economics, Guangzhou, P.R. China

Full list of author information is

available at the end of the article

\begin{abstract}
In this paper, the problem of oscillation for a second-order linear impulsive differential equation with damping is investigated. This equation can be more accurately used to model the states of many evolutionary processes, which are often subject to short-term perturbations and experience abrupt changes at certain moments of time. By employing a generalized Riccati transformation technique, we derive several oscillation criteria which are either new or improve several recent results in the literature. In addition, we provide an example to illustrate the effect of impulses on the oscillation of the equation.
\end{abstract}

Keywords: Oscillation; Impulse; Riccati transformation; Damping; Integral averaging technique

\section{Introduction}

The ordinary differential equation provides a powerful tool to model the continuous processes and phenomena observed in physics, chemistry, population dynamics, biotechnologies, industrial robotics, etc. For example, it can be used to describe some physical, particularly mechanical, phenomena such as the paths of motion of celestial bodies and the brachistochrone problem [5, 7]. Therefore, the dynamics of solution of the equation is paid close attention to by many researchers [9, 17, 19-23]. For example, Sugiyama obtained a singularity formation for the 1D compressible Euler equations with variable damping coefficient [17]. Yang and Ding investigated the longtime dynamics of Boussinesq type equations with fractional damping [23]. The phenomenon of oscillations is observed in physics, economic, etc. Under some conditions, the solutions of the linear differential equation [19], the delay differential equation [5, 7], the linear differential equation with damping [9, 20-22] and the half-linear advanced differential equation [4] would exhibit non-oscillatory or oscillatory properties.

It is well known that motions in the real world are not always continuous processes. In fact, when considering the movement of a mass point, it is sufficiently conceivable that the movement speed of the mass point changes discontinuously by some instantaneous perturbations and experiences abrupt changes at certain moments of time. Those phenomena can be thought of as impulses, therefore, the impulsive differential equation is richer to use to simulate those discontinuous processes being caused by impulses than

(c) The Author(s) 2019. This article is distributed under the terms of the Creative Commons Attribution 4.0 International License (http://creativecommons.org/licenses/by/4.0/), which permits unrestricted use, distribution, and reproduction in any medium, provided you give appropriate credit to the original author(s) and the source, provide a link to the Creative Commons license, and indicate if changes were made. 
the ordinary differential equation [8]. The duration of these changes is very short but it is not negligible for the dynamics of the differential equation, particularly for the oscillatory properties of differential equation. Let us mention some cases. Bohner et al. [3] and Heidarkhani et al. [6] presented new criteria on the existence of three solutions for impulsive boundary-value problems. Shang et al. [15] and Wang [18] obtained a periodic solution for impulsive differential equations. Liu got a new method for converting boundary-value problems of impulsive fractional differential equations to integral equations [10]. Luo and Shen used the associated Riccati techniques and the equivalence transformation to derive the oscillation and the non-oscillation of the second-order linear differential equation with impulses; they indicated that oscillation of impulsive differential equations can be caused by impulsive perturbations, though the corresponding classical equation admitted a non-oscillatory solution [11]. Sugie and Ishihara dealt with the problem of oscillation for a second-order linear differential equation in which an impulsive effect was considered, and they proved that there was a case that the mass point might oscillate due to the influence of the impulsive effect even if the mass point did not oscillate in the model removing the impulsive effect [16]. Ozbekler and Zafer obtained the oscillation criteria of different impulsive differential equations (such as super-half-linear impulsive differential equations [12], half-linear impulsive differential equations [13], and mixed nonlinear differential equations [14]). Agarwal, Karakoc and Zafer provided the methods for studying impulsive differential equations and summarized almost all results up to 2010 on the oscillation of the equations $[1,2]$.

From the above discussions, it is of great significance to take into account the effect of impulses in the investigation of differential equations. The question is whether the nonoscillatory or oscillatory properties of differential equations keep invariant or not considering the effects of both impulses and damping. For this purpose, we devote our efforts to investigating Philos-type oscillation criteria for the second-order linear impulsive differential equation with damping

$$
\left\{\begin{array}{l}
x^{\prime \prime}(t)+p(t) x^{\prime}(t)+q(t) x(t)=0, \quad t \geq t_{0}, t \neq t_{k} \\
\triangle x^{\prime}\left(t_{k}\right)+b_{k} x\left(t_{k}\right)=0
\end{array}\right.
$$

where $p(t), q(t)$ are left piecewise continuous on $\left[t_{0}, \infty\right) ; N$ is a set of positive integers, the sequence of times $\left\{t_{k}\right\}_{k \in N}$ is strictly increasing and satisfies $t_{k} \geq t_{0}$ for some $t_{0}>0$ and $\lim _{k \rightarrow \infty} t_{k}=\infty ; \Delta z\left(t_{k}\right)=z\left(t_{k}^{+}\right)-z\left(t_{k}^{-}\right)$; the coefficient $\left\{b_{k}\right\}$ is a sequence of real numbers.

Definition 1 A function $x$ is said to be a solution of (1) on $\left[t_{0}, \infty\right)$ if

(i) $x(t)$ is continuous on $\left[t_{0}, \infty\right)$,

(ii) $x(t)$ satisfies the first equality of $(1)$ on $\left[t_{0}, \infty\right) \backslash\left\{t_{k}, k \in N\right\}$,

(iii) $x^{\prime}(t)$ has two-side limits and left continuous at points $t_{k}, x^{\prime}\left(t_{k}\right)$ satisfies the second equality of (1).

Definition 2 A nontrivial solution of (1) is said to be non-oscillatory if it is eventually positive or eventually negative. Otherwise, it is said to be oscillatory. Equation (1) is called oscillatory if all nontrivial solutions are oscillatory. 
To formulate the earlier excellent work, we use the following notations. Denote by $t_{k}$ be the moments of impulsive effect for each $k \in N$ and let $N(t)$ be the number of the moments of impulsive effect in the interval $\left[t_{0}, t\right)$, then

$$
N(t)= \begin{cases}0, & \text { if } t_{0} \leq t \leq t_{1} \\ k, & \text { if } t_{k}<t \leq t_{k+1}\end{cases}
$$

The function $p(t)$ of the first equation and the second equation of (1) represent the damping and impulsive effects, respectively. In the case $p(t)=0$, the damping effect disappears and Eq. (1) becomes a second-order linear impulsive differential equation of the form

$$
\left\{\begin{array}{l}
x^{\prime \prime}(t)+q(t) x(t)=0, \quad t \geq t_{0}, t \neq t_{k} \\
\triangle x^{\prime}\left(t_{k}\right)+b_{k} x\left(t_{k}\right)=0
\end{array}\right.
$$

There are many results that have been published on the oscillation of Eq. (3). Here we refer to the latest result given by Sugie and Ishihara [16], we rewrite it as follows.

Theorem A Denote $D=\left\{(t, s): t \geq s \geq t_{0}\right\}$ and let $H: D \rightarrow[0, \infty)$ be a continuous function which satisfies

$$
H(t, t)=0 \quad \text { for } t \geq t_{0}, \quad H(t, s)>0 \quad \text { for } t>s \geq t_{0},
$$

and has a continuous, non-positive partial derivative on $D$ with respect to the second variable s. Moreover, let $h: D \rightarrow[0, \infty)$ be a continuous function with

$$
-\frac{\partial}{\partial s} H(t, s)=h(t, s) \sqrt{H(t, s)} \text { for all }(t, s) \in D
$$

If

$$
\lim _{t \rightarrow \infty} \sup \frac{1}{H\left(t, t_{0}\right)}\left\{\int_{t_{0}}^{t}\left(H(t, s) q(s)-\frac{1}{4} h^{2}(t, s)\right) d s+\sum_{k=1}^{N(t)} H\left(t, t_{k}\right) b_{k}\right\}=\infty
$$

then Eq. (3) is oscillatory.

In the case $b_{k}=0$ for all $k \in N$, which implies that the influence of impulses disappears and Eq. (1) becomes second-order linear differential equation with damping of the form

$$
x^{\prime \prime}(t)+p(t) x^{\prime}(t)+q(t) x(t)=0
$$

which is famous differential equation for describing the forced vibration of an object in physics. We can easily find the results on oscillatory theory for Eq. (5). A representative oscillatory theory is given by Yan [21], we rewrite it as follows.

Theorem B Assume that, for $\alpha>1,0 \leq \beta<1$,

$$
\lim _{t \rightarrow \infty} \sup \frac{1}{t^{\alpha}} \int_{t_{0}}^{t}(t-s)^{\alpha} s^{\beta} q(s) d s=\infty
$$


and

$$
\lim _{t \rightarrow \infty} \sup \frac{1}{t^{\alpha}} \int_{t_{0}}^{t}[(t-s) \operatorname{sp}(s)+\alpha s-\beta(t-s)]^{2}(t-s)^{\alpha-2} s^{\beta-2} d s<\infty
$$

then Eq. (5) is oscillatory.

The aim of this paper is to derive Philos-type oscillatory criteria for a second-order linear impulsive differential equation with damping and to try to illustrate that the oscillation is caused by the damping or the impulses. Our plan is as follows. Using the Riccati transformation and the integral averaging technique, we obtain the oscillation criteria for Eq. (1) in Sect. 2. The results extend and improve the earlier publications. In Sect. 3, choosing two special types of the function $H$, we derive two corollaries by Theorem 1 . In the final section, we illustrate our results with an example, which will show that even if an ordinary differential equation without the impulses has a non-oscillatory solution, on adding the influence of impulses, the equation would be oscillatory. Moreover, the example also shows that the oscillation of the equation is caused by the impulses.

\section{Main results}

Theorem 1 Let $H$ and $h$ be the same functions in Theorem A. Assume that $p(t)>0$ and

$$
\begin{aligned}
& \lim _{t \rightarrow \infty} \sup \frac{1}{H\left(t, t_{0}\right)} \\
& \quad \times\left\{\int_{t_{0}}^{t}\left(H(t, s) q(s)-\frac{1}{4}\left(h^{2}(t, s)+\frac{2 p(s) h(t, s)}{\sqrt{H(t, s)}}+\frac{p^{2}(s)}{H(t, s)}\right)\right) d s\right. \\
& \left.\quad+\sum_{k=1}^{N(t)} H\left(t, t_{k}\right) b_{k}\right\}=\infty,
\end{aligned}
$$

then Eq. (1) is oscillatory.

Proof If (1) has a non-oscillatory solution $x=x(t)$, then there exists a $T \geq t_{0}$ such that $x(t) \neq 0$ for $t \geq T$. Thus, we can define the Riccati transformation as

$$
w(t)=\frac{x^{\prime}(t)}{x(t)} \quad \text { for } t \geq T .
$$

It follows from (1) that

$$
\begin{aligned}
w^{\prime}(t) & =\frac{x^{\prime \prime}(t) x(t)-\left(x^{\prime}(t)\right)^{2}}{x^{2}(t)} \\
& =\frac{x^{\prime \prime}(t)}{x(t)}-\left(\frac{x^{\prime}(t)}{x(t)}\right)^{2} \\
& =\frac{-p(t) x^{\prime}(t)-q(t) x(t)}{x(t)}-\left(\frac{x^{\prime}(t)}{x(t)}\right)^{2} \\
& =-p(t) w(t)-q(t)-w^{2}(t)
\end{aligned}
$$


for $t \neq t_{k}$. Since $x(t)$ is continuous on $[T, \infty)$, it can be seen that

$$
\begin{aligned}
\triangle w\left(t_{k}\right) & =w\left(t_{k}^{+}\right)-w\left(t_{k}^{-}\right) \\
& =\frac{x^{\prime}\left(t_{k}^{+}\right)}{x\left(t_{k}^{+}\right)}-\frac{x^{\prime}\left(t_{k}^{-}\right)}{x\left(t_{k}^{-}\right)} \\
& =\frac{x^{\prime}\left(t_{k}^{+}\right)-x^{\prime}\left(t_{k}^{-}\right)}{x\left(t_{k}\right)} \\
& =\frac{\triangle x^{\prime}\left(t_{k}\right)}{x\left(t_{k}\right)}=-b_{k} .
\end{aligned}
$$

Therefore, the function $w(t)$ satisfies

$$
\left\{\begin{array}{l}
w^{\prime}(t)+w^{2}(t)+p(t) w(t)+q(t)=0, \quad t \neq t_{k} \\
\triangle w\left(t_{k}\right)+b_{k}=0
\end{array}\right.
$$

We choose a positive integer $m$ so that $t_{m-1} \leq T<t_{m}$. For sufficiently large $t$, denote $n=j(t)$. In other words, $n$ is a positive integer which satisfies $t_{n} \leq t<t_{n+1}$. I represents a set of interval $[T, t]$ except the points of $t_{m}, t_{m+1}, \ldots, t_{n}$. It can be seen from the first equation of (9) that

$$
\begin{aligned}
\int_{T}^{t} H(t, s) q(s) d s & =\int_{I} H(t, s) q(s) d s \\
& =-\int_{I} H(t, s) w^{\prime}(s) d s-\int_{I} H(t, s) w^{2}(s) d s-\int_{I} p(s) w(s) d s .
\end{aligned}
$$

Since $w(t)$ is a left piecewise continuous function and $H$ is a continuous function, we see that

$$
\begin{aligned}
H(t, t) w(t)-H(t, T) w(T)= & H(t, t) w(t)-H\left(t, t_{n}^{+}\right) w\left(t_{n}^{+}\right) \\
& +H\left(t, t_{n}^{+}\right) w\left(t_{n}^{+}\right)-H\left(t, t_{n}^{-}\right) w\left(t_{n}^{-}\right) \\
& +H\left(t, t_{n}^{-}\right) w\left(t_{n}^{-}\right)-H\left(t, t_{n-1}^{+}\right) w\left(t_{n-1}^{+}\right) \\
& +H\left(t, t_{n-1}^{+}\right) w\left(t_{n-1}^{+}\right)-H\left(t, t_{n-1}^{-}\right) w\left(t_{n-1}^{-}\right) \\
& +H\left(t, t_{n-1}^{-}\right) w\left(t_{n-1}^{-}\right)-H\left(t, t_{n-2}^{+}\right) w\left(t_{n-2}^{+}\right) \\
& +\cdots+H\left(t, t_{m}^{+}\right) w\left(t_{m}^{+}\right)-H\left(t, t_{m}^{-}\right) w\left(t_{m}^{-}\right) \\
& +H\left(t, t_{m}^{-}\right) w\left(t_{m}^{-}\right)-H(t, T) w(T) \\
= & \int_{I} \frac{\partial}{\partial s}(H(t, s) w(s)) d s \\
& +\sum_{k=m}^{N(t)}\left(H\left(t, t_{k}^{+}\right) w\left(t_{k}^{+}\right)-H\left(t, t_{k}^{-}\right) w\left(t_{k}^{-}\right)\right) \\
= & \int_{I} \frac{\partial}{\partial s}(H(t, s) w(s)) d s \\
& +\sum_{k=m}^{N(t)} H\left(t, t_{k}\right)\left(w\left(t_{k}^{+}\right)-w\left(t_{k}^{-}\right)\right)
\end{aligned}
$$




$$
\begin{aligned}
& =\int_{I} \frac{\partial}{\partial s}(H(t, s) w(s)) d s+\sum_{k=m}^{N(t)} H\left(t, t_{k}\right) \triangle w\left(t_{k}\right) \\
& =\int_{I} \frac{\partial}{\partial s}(H(t, s) w(s)) d s-\sum_{k=m}^{N(t)} H\left(t, t_{k}\right) b_{k} .
\end{aligned}
$$

Owing to the assumption $H(t, t)=0$, it follows from (11) that

$$
\int_{I} \frac{\partial}{\partial s}(H(t, s) w(s)) d s=-H(t, T) w(T)+\sum_{k=m}^{N(t)} H\left(t, t_{k}\right) b_{k} .
$$

Using (10) and (12), and the relationship of $H(t, s)$ and $h(t, s)$, we have

$$
\begin{aligned}
& \int_{I} H(t, s) q(s) d s=-\int_{I}\left(\frac{\partial}{\partial s}(H(t, s) w(s)) d s+\int_{I} \frac{\partial}{\partial s} H(t, s) w(s) d s\right. \\
& -\int_{I} H(t, s) w^{2}(s) d s-\int_{I} p(s) w(s) d s \\
& =H(t, T) w(T)-\sum_{k=m}^{N(t)} H\left(t, t_{k}\right) b_{k}-\int_{I} h(t, s) \sqrt{H(t, s)} w(s) d s \\
& -\int_{I} H(t, s) w^{2}(s) d s-\int_{I} p(s) w(s) d s \\
& =H(t, T) w(T)-\sum_{k=m}^{N(t)} H\left(t, t_{k}\right) b_{k} \\
& -\int_{I}\left(h(t, s) \sqrt{H(t, s)} w(s)+p(s) w(s)+H(t, s) w^{2}(s)\right) d s \\
& =H(t, T) w(T)-\sum_{k=m}^{N(t)} H\left(t, t_{k}\right) b_{k} \\
& -\int_{I} p(s)\left(\left(\frac{h(t, s)}{p(s)} \sqrt{H(t, s)}+1\right) w(s)+\frac{H(t, s)}{p(s)} w^{2}(s)\right) d s \\
& =H(t, T) w(T)-\sum_{k=m}^{N(t)} H\left(t, t_{k}\right) b_{k} \\
& -\int_{I} p(s)\left\{\left(\frac{h(t, s)}{\sqrt{p(s)}}+\frac{\sqrt{p(s)}}{\sqrt{H(t, s)}}\right) \frac{\sqrt{H(t, s)}}{\sqrt{p(s)}} w(s)\right. \\
& \left.+\frac{H(t, s)}{p(s)} w^{2}(s)\right\} d s \\
& =H(t, T) w(T)-\sum_{k=m}^{N(t)} H\left(t, t_{k}\right) b_{k} \\
& -\int_{I} p(s)\left\{-\frac{1}{4}\left(\frac{h(t, s)}{\sqrt{p(s)}}+\frac{\sqrt{p(s)}}{\sqrt{H(t, s)}}\right)^{2}\right. \\
& \left.+\left(\frac{1}{2}\left(\frac{h(t, s)}{\sqrt{p(s)}}+\frac{\sqrt{p(s)}}{\sqrt{H(t, s)}}\right)+\frac{\sqrt{H(t, s)}}{\sqrt{p(s)}} w(s)\right)^{2}\right\} d s
\end{aligned}
$$




$$
\begin{aligned}
= & H(t, T) w(T)-\sum_{k=m}^{N(t)} H\left(t, t_{k}\right) b_{k} \\
& +\frac{1}{4} \int_{I} p(s)\left(\frac{h(t, s)}{\sqrt{p(s)}}+\frac{\sqrt{p(s)}}{\sqrt{H(t, s)}}\right)^{2} d s \\
& -\int_{I} p(s)\left(\frac{1}{2}\left(\frac{h(t, s)}{\sqrt{p(s)}}+\frac{\sqrt{p(s)}}{\sqrt{H(t, s)}}\right)+\frac{\sqrt{H(t, s)}}{\sqrt{p(s)}} w(s)\right)^{2} d s \\
\leq & H(t, T) w(T)-\sum_{k=m}^{N(t)} H\left(t, t_{k}\right) b_{k} \\
& +\frac{1}{4} \int_{I} p(s)\left(\frac{h(t, s)}{\sqrt{p(s)}}+\frac{\sqrt{p(s)}}{\sqrt{H(t, s)}}\right)^{2} d s .
\end{aligned}
$$

It turns out that

$$
\begin{gathered}
\int_{I} H(t, s) q(s) d s-\frac{1}{4} \int_{I} p(s)\left(\frac{h(t, s)}{\sqrt{p(s)}}+\frac{\sqrt{p(s)}}{\sqrt{H(t, s)}}\right)^{2} d s \\
+\sum_{k=m}^{N(t)} H\left(t, t_{k}\right) b_{k} \leq H(t, T) w(T) .
\end{gathered}
$$

That is,

$$
\begin{aligned}
& \int_{I}\left(H(t, s) q(s)-\frac{1}{4}\left(h^{2}(t, s)+\frac{2 p(s) h(t, s)}{\sqrt{H(t, s)}}+\frac{p^{2}(s)}{H(t, s)}\right)\right) d s \\
& \quad+\sum_{k=m}^{N(t)} H\left(t, t_{k}\right) b_{k} \leq H(t, T) w(T) .
\end{aligned}
$$

Therefore, we obtain

$$
\begin{aligned}
& \int_{t_{0}}^{t}\left(H(t, s) q(s)-\frac{1}{4}\left(h^{2}(t, s)+\frac{2 p(s) h(t, s)}{\sqrt{H(t, s)}}+\frac{p^{2}(s)}{H(t, s)}\right)\right) d s+\sum_{k=1}^{N(t)} H\left(t, t_{k}\right) b_{k} \\
& =\int_{t_{0}}^{T}\left(H(t, s) q(s)-\frac{1}{4}\left(h^{2}(t, s)+\frac{2 p(s) h(t, s)}{\sqrt{H(t, s)}}+\frac{p^{2}(s)}{H(t, s)}\right)\right) d s+\sum_{k=1}^{m-1} H\left(t, t_{k}\right) b_{k} \\
& \quad+\int_{T}^{t}\left(H(t, s) q(s)-\frac{1}{4}\left(h^{2}(t, s)+\frac{2 p(s) h(t, s)}{\sqrt{H(t, s)}}+\frac{p^{2}(s)}{H(t, s)}\right)\right) d s+\sum_{k=m}^{N(t)} H\left(t, t_{k}\right) b_{k} \\
& \leq \int_{t_{0}}^{T}\left(H(t, s) q(s)-\frac{1}{4}\left(h^{2}(t, s)+\frac{2 p(s) h(t, s)}{\sqrt{H(t, s)}}+\frac{p^{2}(s)}{H(t, s)}\right)\right) d s \\
& \quad+\sum_{k=1}^{m-1} H\left(t, t_{k}\right) b_{k}+H(t, T) w(T) .
\end{aligned}
$$


Since $p(t)>0$ and $H(t, s)$ is decreasing with respect to the second variable $s$, we see that

$$
\begin{aligned}
\int_{t_{0}}^{t}\left(H(t, s) q(s)-\frac{1}{4}\left(h^{2}(t, s)+\frac{2 p(s) h(t, s)}{\sqrt{H(t, s)}}+\frac{p^{2}(s)}{H(t, s)}\right)\right) d s \\
\quad+\sum_{k=1}^{N(t)} H\left(t, t_{k}\right) b_{k} \\
\leq \int_{t_{0}}^{T}\left(H(t, s) q(s)-\frac{1}{4}\left(h^{2}(t, s)+\frac{2 p(s) h(t, s)}{\sqrt{H(t, s)}}+\frac{p^{2}(s)}{H(t, s)}\right)\right) d s \\
\quad+\sum_{k=1}^{m-1} H\left(t, t_{k}\right) b_{k}+H(t, T) w(T) \\
=\int_{t_{0}}^{T}\left(H(t, s) q(s)-\frac{1}{4} p(s)\left(\frac{h(t, s)}{\sqrt{p(s)}}+\frac{\sqrt{p(s)}}{\sqrt{H(t, s)}}\right)^{2}\right) d s \\
\quad+\sum_{k=1}^{m-1} H\left(t, t_{k}\right) b_{k}+H(t, T) w(T) \\
\leq \int_{t_{0}}^{T} H(t, s) q(s) d s+\sum_{k=1}^{m-1} H\left(t, t_{k}\right) b_{k}+H(t, T) w(T) \\
\leq \int_{t_{0}}^{T} H(t, s)|q(s)| d s+\sum_{k=1}^{m-1} H\left(t, t_{k}\right)\left|b_{k}\right|+H(t, T)|w(T)| \\
\quad H\left(t_{0}\right)\left(\int_{t_{0}}^{T}|q(s)| d s+\sum_{k=1}^{m-1}\left|b_{k}\right|+|w(T)|\right)
\end{aligned}
$$

Hence, we conclude that

$$
\begin{aligned}
& \lim _{t \rightarrow \infty} \sup \frac{1}{H\left(t, t_{0}\right)} \\
& \quad \times\left\{\int_{t_{0}}^{t}\left(H(t, s) q(s)-\frac{1}{4}\left(h^{2}(t, s)+\frac{2 p(s) h(t, s)}{\sqrt{H(t, s)}}+\frac{p^{2}(s)}{H(t, s)}\right)\right) d s\right. \\
& \left.\quad+\sum_{k=1}^{N(t)} H\left(t, t_{k}\right) b_{k}\right\}<\infty,
\end{aligned}
$$

which contradicts the condition (8). The proof of Theorem 1 is complete.

Remark 1 When $p(t)=0$, Eq. (1) reduces to Eq. (3), Theorem 1 is a natural extension of Theorem A given by Sugie and Ishihara [16].

It can be seen that we constrain the damped term $p(t)>0$ in the above results. If the sign of $p(t)$ is allowed to change, we derive the following result.

Theorem 2 Assume that, for $\alpha>1,0 \leq \beta<1$,

$$
\lim _{t \rightarrow \infty} \sup \frac{1}{t^{\alpha}}\left\{\int_{t_{0}}^{t}\left((t-s)^{\alpha} s^{\beta} q(s)\right) d s+\sum_{k=1}^{N(t)}\left(t-t_{k}\right)^{\alpha} b_{k}\right\}=\infty
$$


and

$$
\lim _{t \rightarrow \infty} \sup \frac{1}{t^{\alpha}} \int_{t_{0}}^{t}[(t-s) \operatorname{sp}(s)+\alpha s-\beta(t-s)]^{2}(t-s)^{\alpha-2} s^{\beta-2} d s<\infty
$$

then Eq. (1) is oscillatory.

Proof If Eq. (1) has a non-oscillatory solution $x=x(t)$, then there exists a $T \geq t_{0}$ such that $x(t) \neq 0$ for $t \geq T$. Thus, we can define the Riccati transformation as

$$
w(t)=\frac{x^{\prime}(t)}{x(t)} \quad \text { for } t \geq T
$$

Therefore, the function $w(t)$ satisfies (9)

$$
\left\{\begin{array}{l}
w^{\prime}(t)+w^{2}(t)+p(t) w(t)+q(t)=0, \quad t \neq t_{k} \\
\triangle w\left(t_{k}\right)+b_{k}=0
\end{array}\right.
$$

Multiplying the first equality of (9) with $(t-s)^{\alpha} s^{\beta}$ and integrating it from $t_{0}$ to $t$, we have

$$
\begin{aligned}
\int_{t_{0}}^{t}(t-s)^{\alpha} s^{\beta} q(s) d s= & -\int_{t_{0}}^{t}(t-s)^{\alpha} s^{\beta} w^{\prime}(s) d s-\int_{t_{0}}^{t}(t-s)^{\alpha} s^{\beta} w^{2}(s) d s \\
& -\int_{t_{0}}^{t}(t-s)^{\alpha} s^{\beta} p(s) w(s) d s,
\end{aligned}
$$

note that

$$
\begin{aligned}
-\int_{t_{0}}^{t}(t-s)^{\alpha} s^{\beta} w^{\prime}(s) d s= & \left(t-t_{0}\right)^{\alpha} t_{0}^{\beta} w\left(t_{0}\right) \\
& -\sum_{k=1}^{N(t)}\left(t-t_{k}\right)^{\alpha} t_{k}^{\beta} b_{k}-\alpha \int_{t_{0}}^{t}(t-s)^{\alpha-1} s^{\beta} w(s) d s \\
& +\beta \int_{t_{0}}^{t}(t-s)^{\alpha} s^{\beta-1} w(s) d s
\end{aligned}
$$

it follows from (16) and (17) that

$$
\begin{aligned}
& \int_{t_{0}}^{t}(t-s)^{\alpha} s^{\beta} q(s) d s \\
& =\left(t-t_{0}\right)^{\alpha} t_{0}^{\beta} w\left(t_{0}\right)-\sum_{k=1}^{N(t)}\left(t-t_{k}\right)^{\alpha} t_{k}^{\beta} b_{k} \\
& \quad-\alpha \int_{t_{0}}^{t}(t-s)^{\alpha-1} s^{\beta} w(s) d s+\beta \int_{t_{0}}^{t}(t-s)^{\alpha} s^{\beta-1} w(s) d s \\
& \quad-\int_{t_{0}}^{t}(t-s)^{\alpha} s^{\beta} w^{2}(s) d s-\int_{t_{0}}^{t}(t-s)^{\alpha} s^{\beta} p(s) w(s) d s \\
& =\left(t-t_{0}\right)^{\alpha} t_{0}^{\beta} w\left(t_{0}\right)-\sum_{k=1}^{N(t)}\left(t-t_{k}\right)^{\alpha} t_{k}^{\beta} b_{k}
\end{aligned}
$$




$$
\begin{aligned}
& -\int_{t_{0}}^{t}[(t-s) \operatorname{sp}(s)+\alpha s-\beta(t-s)](t-s)^{\alpha-1} s^{\beta-1} w(s) d s \\
& -\int_{t_{0}}^{t}(t-s)^{\alpha} s^{\beta} w^{2}(s) d s \\
= & \left(t-t_{0}\right)^{\alpha} t_{0}^{\beta} w\left(t_{0}\right)-\sum_{k=1}^{N(t)}\left(t-t_{k}\right)^{\alpha} t_{k}^{\beta} b_{k} \\
& +\frac{1}{4} \int_{t_{0}}^{t}[(t-s) \operatorname{sp}(s)+\alpha s-\beta(t-s)]^{2}(t-s)^{\alpha-2} s^{\beta-2} d s \\
& -\int_{t_{0}}^{t}\left\{(t-s)^{\frac{\alpha}{2}} s^{\frac{\beta}{2}} w(s)\right. \\
& \left.+\frac{1}{2}[(t-s) \operatorname{sp}(s)+\alpha s-\beta(t-s)](t-s)^{\frac{\alpha-2}{2}} s^{\frac{\beta-2}{2}}\right\}^{2} d s \\
\leq & \left(t-t_{0}\right)^{\alpha} t_{0}^{\beta} w\left(t_{0}\right)-\sum_{k=1}^{N(t)}\left(t-t_{k}\right)^{\alpha} t_{k}^{\beta} b_{k} \\
& +\frac{1}{4} \int_{t_{0}}^{t}[(t-s) \operatorname{sp}(s)+\alpha s-\beta(t-s)]^{2}(t-s)^{\alpha-2} s^{\beta-2} d s .
\end{aligned}
$$

Hence, we get

$$
\begin{aligned}
& \int_{t_{0}}^{t}(t-s)^{\alpha} s^{\beta} q(s) d s+\sum_{k=1}^{N(t)}\left(t-t_{k}\right)^{\alpha} t_{k}^{\beta} b_{k} \\
& \leq\left(t-t_{0}\right)^{\alpha} t_{0}^{\beta} w\left(t_{0}\right) \\
& \quad+\frac{1}{4} \int_{t_{0}}^{t}[(t-s) \operatorname{sp}(s)+\alpha s-\beta(t-s)]^{2}(t-s)^{\alpha-2} s^{\beta-2} d s .
\end{aligned}
$$

Dividing (19) by $t^{\alpha}$ and using (14), we conclude that

$$
\begin{aligned}
& \limsup _{t \rightarrow \infty} \frac{1}{t^{\alpha}}\left\{\int_{t_{0}}^{t}(t-s)^{\alpha} s^{\beta} q(s) d s+\sum_{k=1}^{N(t)}\left(t-t_{k}\right)^{\alpha} t_{k}^{\beta} b_{k}\right\} \\
& \leq \limsup _{t \rightarrow \infty} \frac{\left(t-t_{0}\right)^{\alpha} t_{0}^{\beta} w\left(t_{0}\right)}{t^{\alpha}} \\
& \quad+\frac{1}{4} \limsup _{t \rightarrow \infty} \frac{1}{t^{\alpha}} \int_{t_{0}}^{t}[(t-s) \operatorname{sp}(s)+\alpha s-\beta(t-s)]^{2}(t-s)^{\alpha-2} s^{\beta-2} d s \\
& =t_{0}^{\beta} w\left(t_{0}\right)+\frac{1}{4} \limsup _{t \rightarrow \infty} \frac{1}{t^{\alpha}} \int_{t_{0}}^{t}[(t-s) \operatorname{sp}(s)+\alpha s-\beta(t-s)]^{2}(t-s)^{\alpha-2} s^{\beta-2} d s \\
& <\infty
\end{aligned}
$$

which contradicts (13). The proof of Theorem 2 is complete.

Remark 2 If the influence of impulses is removed, Eq. (1) reduces to Eq. (5), then Theorem 2 extends and improves Theorem B given by Yan [21]. 


\section{Two Corollaries}

In Theorem 1, if we choose the function $H$ as

$$
H(t, s)=(t-s)^{\alpha}, \quad \alpha>1,
$$

then we can obtain the following result.

Corollary 3 Assume that $p(t)>0$ and

$$
\begin{aligned}
& \lim _{t \rightarrow \infty} \sup \frac{1}{t^{\alpha}}\left\{\int_{t_{0}}^{t}\left((t-s)^{\alpha} q(s)-\frac{1}{4}\left(\frac{2 p(s) h(t, s)}{(t-s)^{\frac{\alpha}{2}}}+\frac{p^{2}(s)}{(t-s)^{\alpha}}\right)\right) d s\right. \\
& \left.+\sum_{k=1}^{N(t)}\left(t-t_{k}\right)^{\alpha} b_{k}\right\}=\infty,
\end{aligned}
$$

where $\alpha>1, h(t, s)=\alpha(t-s)^{\frac{\alpha-2}{2}}$, then Eq. (1) is oscillatory.

Proof For any $\alpha>1$, choose

$$
H(t, s)=(t-s)^{\alpha},
$$

then the function $h$ can be defined by

$$
h(t, s)=\alpha(t-s)^{\frac{\alpha-2}{2}} .
$$

It can be seen that the functions $H$ and $h$ satisfy the conditions (A) and (B) in Theorem 1. Moreover,

$$
\begin{aligned}
\frac{1}{H\left(t, t_{0}\right)} \int_{t_{0}}^{t} h^{2}(t, s) d s & =\frac{\alpha^{2}}{\left(t-t_{0}\right)^{\alpha}} \int_{t_{0}}^{t}(t-s)^{\alpha-2} d s \\
& =\frac{\alpha^{2}}{(\alpha-1)\left(t-t_{0}\right)} \longrightarrow 0 \quad(\text { as } t \longrightarrow \infty)
\end{aligned}
$$

and

$$
\frac{t^{\alpha}}{H\left(t, t_{0}\right)}=\frac{t^{\alpha}}{\left(t-t_{0}\right)^{\alpha}}=\left(\frac{1}{1-\frac{t_{0}}{t}}\right)^{\alpha} \rightarrow 1 \quad(\text { as } t \rightarrow \infty) .
$$

Therefore, we conclude that

$$
\begin{aligned}
\lim _{t \rightarrow \infty} & \sup \frac{1}{H\left(t, t_{0}\right)} \\
& \times\left\{\int_{t_{0}}^{t}\left(H(t, s) q(s)-\frac{1}{4}\left(h^{2}(t, s)+\frac{2 p(s) h(t, s)}{\sqrt{H(t, s)}}+\frac{p^{2}(s)}{H(t, s)}\right)\right) d s\right. \\
& \left.+\sum_{k=1}^{N(t)} H\left(t, t_{k}\right) b_{k}\right\}
\end{aligned}
$$




$$
\begin{aligned}
= & \lim _{t \rightarrow \infty} \sup \frac{1}{t^{\alpha}}\left\{\int_{t_{0}}^{t}\left((t-s)^{\alpha} q(s)-\frac{1}{4}\left(\frac{2 p(s) h(t, s)}{(t-s)^{\frac{\alpha}{2}}}+\frac{p^{2}(s)}{(t-s)^{\alpha}}\right)\right) d s\right. \\
& \left.+\sum_{k=1}^{N(t)}\left(t-t_{k}\right)^{\alpha} b_{k}\right\}=\infty .
\end{aligned}
$$

By Theorem 1, Eq. (1) is oscillatory.

For any positive integer $n \geq 2$, if we choose the function $H$ as

$$
H(t, s)=\left(\ln \frac{t}{s}\right)^{n}
$$

respectively, the function $h$ can be defined as

$$
h(t, s)=\frac{n}{s}\left(\ln \frac{t}{s}\right)^{\frac{n-2}{2}}
$$

Similarly to the proof of Corollary 3, we can obtain the following result.

Corollary 4 Assume that $p(t)>0$ and

$$
\begin{aligned}
& \lim _{t \rightarrow \infty} \sup \frac{1}{(\ln t)^{n}} \\
& \quad \times\left\{\int_{t_{0}}^{t}\left(\left(\ln \frac{t}{s}\right)^{n} q(s)-\frac{1}{4}\left[2 p(s) h(t, s)\left(\ln \frac{t}{s}\right)^{-\frac{n}{2}}+p^{2}(s)\left(\ln \frac{t}{s}\right)^{-n}\right]\right) d s\right. \\
& \left.\quad+\sum_{k=1}^{N(t)}\left(\ln \frac{t}{t_{k}}\right)^{n} b_{k}\right\}=\infty,
\end{aligned}
$$

where positive integer $n \geq 2, h(t, s)=\frac{n}{s}\left(\ln \frac{t}{s}\right)^{\frac{n-2}{2}}$, then Eq. (1) is oscillatory.

Remark 3 When $p(t)=0$, Eq. (1) reduces to Eq. (3), our Corollary 3 and Corollary 4 are also generalizations of the corresponding corollaries given by Sugie and Ishihara [16]. When not considering the effect of impulses, our Corollary 3 and Corollary 4 are still new for Eq. (5).

\section{Examples}

Consider the equation

$$
\left\{\begin{array}{l}
x^{\prime \prime}(t)+\frac{A}{t} x^{\prime}(t)+\frac{B}{t^{2}} x(t)=0, \quad t \geq \frac{1}{2}, t \neq k \\
\triangle x^{\prime}(k)+\frac{1}{k} x(k)=0
\end{array}\right.
$$

where $t_{0}=\frac{1}{2}, t_{k}=k, b_{k}=\frac{1}{k}, p(t)=\frac{A}{t}, q(t)=\frac{B}{t^{2}}$ with $A>0, B \geq 0$ and $(A-1)^{2}=4 B$. Letting $n=2$, we calculate that

$$
\begin{aligned}
\frac{1}{(\ln t)^{2}} \int_{t_{0}}^{t}\left(\ln \frac{t}{s}\right)^{2} q(s) d s & =\frac{B}{(\ln t)^{2}} \int_{\frac{1}{2}}^{t} \frac{(\ln t-\ln s)^{2}}{s^{2}} d s \\
& =B\left\{2-\frac{4\left(1+\ln \left(\frac{1}{2}\right)\right)}{\ln t}-\frac{2}{t(\ln t)^{2}}+\frac{4\left(1+\ln \left(\frac{1}{2}\right)\right)+2\left(\ln \left(\frac{1}{2}\right)\right)^{2}}{(\ln t)^{2}}\right\}
\end{aligned}
$$


thus, we have

$$
\lim _{t \rightarrow \infty} \sup \frac{1}{(\ln t)^{2}} \int_{t_{0}}^{t}\left(\ln \frac{t}{s}\right)^{2} q(s) d s \leq 2 B .
$$

Also, since

$$
\frac{-1}{4(\ln t)^{2}} \int_{t_{0}}^{t} p(s) h(t, s)\left(\ln \frac{t}{s}\right)^{-1} d s=\frac{-A}{(\ln t)^{2}} \int_{\frac{1}{2}}^{t} \frac{1}{s^{2} \ln \frac{t}{s}} d s
$$

letting $\tau=\frac{t}{s}$, we have

$$
\begin{aligned}
\frac{-A}{(\ln t)^{2}} \int_{\frac{1}{2}}^{t} \frac{1}{s^{2} \ln \frac{t}{s}} d s & =\frac{-A}{(\ln t)^{2}} \int_{1}^{2 t} \frac{1}{t \ln \tau} d \tau \\
& \leq \frac{-A}{t(\ln t)^{2}} \int_{1}^{2 t} \frac{1}{\ln 2 t} d \tau \\
& =\frac{-A(2 t-1)}{t(\ln t)^{2} \ln 2 t}
\end{aligned}
$$

then

$$
\lim _{t \rightarrow \infty} \sup \frac{-1}{4(\ln t)^{2}} \int_{t_{0}}^{t} p(s) h(t, s)\left(\ln \frac{t}{s}\right)^{-1} d s=0 .
$$

Similarly, we also can get

$$
\lim _{t \rightarrow \infty} \sup \frac{-1}{4(\ln t)^{2}} \int_{t_{0}}^{t} p^{2}(s)\left(\ln \frac{t}{s}\right)^{-2} d s=0 .
$$

Next, we evaluate the effect of the impulse. Based on the definition of $N(t)$, for positive integer $k$ with $m<t<m+1$, we have

$$
N(t)= \begin{cases}0 & \text { for } t_{0} \leq t \leq t_{1} \\ m & \text { for } t_{m}<t \leq t_{m+1}\end{cases}
$$

Furthermore,

$$
\begin{aligned}
\frac{1}{(\ln t)^{2}} \sum_{k=1}^{N(t)}\left(\ln \frac{t}{t_{k}}\right)^{2} b_{k} & =\frac{1}{(\ln t)^{2}} \sum_{k=1}^{m}(\ln t-\ln k)^{2} \frac{1}{k} \\
& =\sum_{k=1}^{m}\left(1-\frac{2 \ln k}{\ln t}+\frac{(\ln k)^{2}}{(\ln t)^{2}}\right) \frac{1}{k} \\
& >\sum_{k=1}^{m}\left(1-\frac{2 \ln k}{\ln m}+\frac{(\ln k)^{2}}{(\ln (m+1))^{2}}\right) \frac{1}{k} \\
& =\sum_{k=1}^{m} \frac{1}{k}-\frac{2}{\ln m} \sum_{k=1}^{m} \frac{\ln k}{k}+\frac{1}{(\ln (m+1))^{2}} \sum_{k=1}^{m} \frac{(\ln k)^{2}}{k} .
\end{aligned}
$$


It follows from $k<s<k+1$ that

$$
\begin{aligned}
\sum_{k=1}^{m} \frac{1}{k} & =1+\frac{1}{2}+\frac{1}{3}+\frac{1}{4}+\cdots+\frac{1}{m} \\
& =\int_{1}^{2} 1 d s+\int_{2}^{3} \frac{1}{2} d s+\int_{3}^{4} \frac{1}{3} d s+\cdots+\int_{m}^{m+1} \frac{1}{m} d s \\
> & \int_{1}^{2} \frac{1}{s} d s+\int_{2}^{3} \frac{1}{s} d s+\int_{3}^{4} \frac{1}{s} d s+\cdots+\int_{m}^{m+1} \frac{1}{s} d s \\
= & \int_{1}^{m+1} \frac{1}{s} d s=\ln (m+1), \\
\sum_{k=1}^{m} \frac{\ln k}{k} & =\frac{\ln 2}{2}+\frac{\ln 3}{3}+\frac{\ln 4}{4}+\cdots+\frac{\ln m}{m} \\
& =\frac{\ln 2}{2}+\frac{\ln 3}{3}+\int_{3}^{4} \frac{\ln 4}{4} d s+\cdots+\int_{m-1}^{m} \frac{\ln m}{m} d s \\
& <\frac{\ln 2}{2}+\frac{\ln 3}{3}+\int_{3}^{4} \frac{\ln s}{s} d s+\cdots+\int_{m-1}^{m} \frac{\ln s}{s} d s \\
& =\frac{\ln 2}{2}+\frac{\ln 3}{3}+\int_{3}^{m} \frac{\ln s}{s} d s \\
& \frac{\ln 3}{3}+\frac{(\ln m)^{2}}{2}-\frac{(\ln 3)^{2}}{2},
\end{aligned}
$$

and

$$
\sum_{k=1}^{m} \frac{(\ln k)^{2}}{k}>\frac{(\ln 2)^{2}}{2}+\frac{(\ln 3)^{2}}{3}+\cdots+\frac{(\ln 7)^{2}}{7}+\frac{(\ln (m+1))^{3}}{3}-\frac{(\ln 8)^{3}}{3}
$$

then

$$
\begin{aligned}
\frac{1}{(\ln t)^{2}} \sum_{k=1}^{N(t)}\left(\ln \frac{t}{t_{k}}\right)^{2} b_{k}> & \ln (m+1)-\frac{2}{\ln m}\left(\frac{\ln 2}{2}+\frac{\ln 3}{3}+\frac{(\ln m)^{2}}{2}-\frac{(\ln 3)^{2}}{2}\right) \\
& +\frac{1}{(\ln (m+1))^{2}}\left(\frac{(\ln 2)^{2}}{2}+\frac{(\ln 3)^{2}}{3}+\cdots\right. \\
& \left.+\frac{(\ln 7)^{2}}{7}+\frac{(\ln (m+1))^{3}}{3}-\frac{(\ln 8)^{3}}{3}\right) \\
= & \frac{4}{3} \ln (m+1)-\ln m-\frac{2}{\ln m}\left(\frac{\ln 2}{2}+\frac{\ln 3}{3}-\frac{(\ln 3)^{2}}{2}\right) \\
& +\frac{1}{(\ln (m+1))^{2}}\left(\frac{(\ln 2)^{2}}{2}+\frac{(\ln 3)^{2}}{3}+\cdots\right. \\
& \left.+\frac{(\ln 7)^{2}}{7}-\frac{(\ln 8)^{3}}{3}\right) \\
= & \ln \left\{\left(1+\frac{1}{m}\right)^{\frac{4}{3}} m^{\frac{1}{3}}\right\}-\frac{2}{\ln m}\left(\frac{\ln 2}{2}+\frac{\ln 3}{3}-\frac{(\ln 3)^{2}}{2}\right) \\
& +\frac{1}{(\ln (m+1))^{2}}\left(\frac{(\ln 2)^{2}}{2}+\frac{(\ln 3)^{2}}{3}+\cdots+\frac{(\ln 7)^{2}}{7}-\frac{(\ln 8)^{3}}{3}\right),
\end{aligned}
$$


letting $m \longrightarrow \infty$, we have

$$
\lim _{t \rightarrow \infty} \sup \frac{1}{(\ln t)^{2}} \sum_{k=1}^{N(t)}\left(\ln \frac{t}{t_{k}}\right)^{2} b_{k}=\infty .
$$

Therefore, the condition (23) holds in the case of $n=2$. By Corollary 4, Eq. (1) is oscillatory. On the other hand, if the effect of impulse is removed and $A>0, B \geq 0$ and $(A-1)^{2}=4 B$, then Eq. (24) is

$$
x^{\prime \prime}(t)+\frac{A}{t} x^{\prime}(t)+\frac{B}{t^{2}} x(t)=0,
$$

and it has a non-oscillatory solution

$$
x(t)=t^{\frac{1-A}{2}} .
$$

Remark 4 It can be seen from the example that the oscillation of Eq. (24) is entirely caused by the impulses.

\section{Acknowledgements}

The authors wish to express their sincere thanks to the anonymous referees and the handling editor for many constructive comments leading to the improved version of this paper.

\section{Funding}

This work was supported by the Natural Science Foundation of China (11701117), Key Research Platform and Research Project of Universities in Guangdong Province (2018KQNCX244) and the Natural Science Foundation of Guangdong Province (2017A030310590 and 2018A030307024)

\section{Availability of data and materials}

Data sharing not applicable to this article as no data sets were generated or analyzed during the current study.

\section{Competing interests}

The authors declare that they have no competing interests.

\section{Authors' contributions}

All authors contributed equally to the writing of this paper. All authors read and approved the final manuscript.

\section{Author details}

${ }^{1}$ School of Mathematics, Jiaying University, Meizhou, P.R. China. ${ }^{2}$ College of Mathematics and Statistics, Guangxi Normal University, Guilin, P.R. China. ${ }^{3}$ School of Statistics and Mathematics, Guangdong University of Finance and Economics, Guangzhou, P.R. China.

\section{Publisher's Note}

Springer Nature remains neutral with regard to jurisdictional claims in published maps and institutional affiliations.

Received: 28 December 2018 Accepted: 11 June 2019 Published online: 24 June 2019

\section{References}

1. Agarwal, R.P., Karakoc, F.: A survey on oscillation of impulsive delay differential equations. Comput. Math. Appl. 60 1648-1685 (2010)

2. Agarwal, R.P., Karakoc, F., Zafer, A.: A survey on oscillation of impulsive ordinary differential equations. Adv. Differ. Equ. 2010, 354841 (2010). https://doi.org/10.1155/2010/354841

3. Bohner, M., Heidarkhani, S., Salari, A., Caristi, G.: Existence of three solutions for impulsive multi-point boundary value problems. Opusc. Math. 37(3), 353-379 (2017)

4. Chatzarakis, G.E., Džurina, J., Jadlovská, I.: New oscillation criteria for second-order half-linear advanced differential equations. Appl. Math. Comput. 347, 404-416 (2019)

5. Hartman, P.: Ordinary Differential Equations. Classics in Appl. Math., vol. 38. Society for Industrial and Applied Mathematics, Philadelphia (2002) Corrected reprint of the second (1982) edition, with a foreword by Peter Bates

6. Heidarkhani, S., Ferrara, M., Caristi, G., Salari, A.: Existence of three solutions for impulsive nonlinear fractional boundary value problems. Opusc. Math. 37(2), 281-301 (2017) 
7. Kelley, W., Peterson, A.: The Theory of Differential Equations Classical and Qualitative. Pearson Education, New Jersey (2004)

8. Lakshmikantham, V., Bainov, D.D., Simeonov, P.S.: Theory of Impulsive Differential Equations. World Scientific, Singapore (1989)

9. Liu, W., Li, H.: Oscillation criteria for second order linear differential equations with damping. J. Appl. Anal. 2, 105-118 (1996)

10. Liu, Y: A new method for converting boundary value problems for impulsive fractional differential equations to integral equations and its applications. Adv. Nonlinear Anal. 8(1), 386-454 (2019)

11. Luo, Z., Shen, J.: Oscillation of second order linear differential equations with impulses. Appl. Math. Lett. 20,75-81 (2007)

12. Özbekler, A., Zafer, A.: Forced oscillation of super-half-linear impulsive differential equations. Comput. Math. Appl. 54 785-792 (2007)

13. Özbekler, A., Zafer, A.: Leighton-Coles-Wintner type oscillation criteria half-linear impulsive differential equations. Adv. Dyn. Syst. Appl. 5, 205-214 (2010)

14. Özbekler, A., Zafer, A.: Oscillation of solutions of second order mixed nonlinear differential equations under impulsive perturbations. Comput. Math. Appl. 61, 933-940 (2011)

15. Shang, S., Bai, Z., Tian, Y., Yue, Y.: Periodic solution for second-order impulsive differential inclusions with relativistic operator. Bound. Value Probl. 2018, 173 (2018)

16. Sugie, J., Ishihara, K.: Philostype oscillation criteria for linear differential equations with impulsive effects. J. Math. Anal. Appl. 470, 911-930 (2019)

17. Sugiyama, $Y$ : Singularity formation for the $1 \mathrm{D}$ compressible Euler equations with variable damping coefficient. Nonlinear Anal. 170, 70-87 (2018)

18. Wang, S.: The existence of affine-periodic solutions for nonlinear impulsive differential equations. Bound. Value Probl. 2018, $113(2018)$

19. Willett, D.W.: On the oscillatory behavior of the solutions of second order linear differential equations. Ann. Pol. Math. 21, 175-194 (1969)

20. Wong, J.: On Kamenev-type oscillation theorems for second-order differential equations with damping. J. Math. Anal. Appl. 258, 244-257 (2001)

21. Yan, J: A note on an oscillation criterion for an equation with damped term. Proc. Am. Math. Soc. 90, 277-280 (1984)

22. Yan, J.: Oscillation theorems for second order linear differential equations with damping. Proc. Am. Math. Soc. 90, 276-282 (1986)

23. Yang, Z., Ding, P.: Longtime dynamics of Boussinesq type equations with fractional damping. Nonlinear Anal. 161 108-130 (2017)

\section{Submit your manuscript to a SpringerOpen ${ }^{\circ}$ journal and benefit from:}

- Convenient online submission

- Rigorous peer review

- Open access: articles freely available online

- High visibility within the field

- Retaining the copyright to your article

Submit your next manuscript at $\boldsymbol{\nabla}$ springeropen.com 